\title{
Relación entre el ángulo de la base craneal y las clases esqueletales en una población adulta de Paraguay
}

\author{
Relationship Between Cranial Base Angle \\ and Skeletal Classes in an Adult Population of \\ Paraguay
}

\section{Relação entre o ângulo da base craniano e as classes esqueléticas em uma população adulta do Paraguai}

Cristell Amarilla, Esp ${ }^{1}$

Clara Maldonado Renault, Esp ${ }^{1}$

Leticia Galeano, Esp ${ }^{1}$

Julio Pratt Giosa, Esp²

Clarisse Díaz-Reissner, $\mathrm{PhD}^{2}$

Recibido: 18 de junio de 2019 - Aceptado: 25 de octubre de 2019

Doi: https://doi.org/10.12804/revistas.urosario.edu.co/revsalud/a.8767

Para citar este artículo: Amarilla C, Maldonado Renault C, Galeano L, Pratt Giosa J, Díaz-Reissner C. Relación entre el ángulo de la base craneal y las clases esqueletales en una población adulta de Paraguay. Rev Cienc Salud. 2020;18(1):96-107. https://doi.org/10.12804/ revistas.urosario.edu.co/revsalud/a.8767

\section{Resumen}

Introducción: numerosos estudios mencionan que las variaciones del crecimiento y orientación del ángulo de la base craneal pueden alterar la posición de ambos maxilares en relación con la base craneal. En este estudio se busca relacionar el ángulo de la base craneal con las clases esqueletales, en telerradiografías laterales de pacientes adultos que acudieron a la Clínica Odontológica del Instituto Latinoamericano de Estudios Superiores, de la ciudad de Asunción, Paraguay, entre los años 2010 y 2018. Materiales y métodos: estudio transversal retrospectivo. Para clasificar la clase esqueletal, se utilizó el ángulo ANB de Steiner, y para determinar el ángulo de la base craneal posterior, el NSBA de Varjanne y Koski. Luego, se aplicó la prueba chi-cuadrado para relacionar el ángulo de la base craneal y las clases esqueletales, con un nivel de confianza del 95\%. Resultados: formaron parte del estudio 185 telerradiografías laterales de cráneo de pacientes que acudieron a la clínica. La media del ángulo NSBa fue

1 Posgrado en Ortodoncia Correctiva, Instituto Latinoamericano de Estudios Superiores, Paraguay.

2 Dirección de Investigación, Facultad de Odontología, Universidad Nacional de Asunción, Paraguay. Autor de correspondencia: cdiazr@odo.una.py 
$128.4^{\circ} \pm 5.17^{\circ}$; la clase I fue de $128.27^{\circ} \pm 5.27^{\circ}$; la clase II, de $129.1^{\circ} \pm 4.7^{\circ}$; y la clase III, de $127.84^{\circ} \pm 5^{\circ}$. El $65.95 \%$ posee valores del ángulo NSBa fuera de la norma, de los cuales el $87.7 \%$ presentan un ángulo cerrado. Conclusión: el promedio del ángulo de la base craneal estaba fuera de la norma en todas las clases esqueletales, siendo más frecuente la flexión craneal, en orden decreciente para las clases III, I y II.

Palabras claves: ortodoncia; base del cráneo; diagnóstico por imagen; cefalometría; Paraguay

Abstract

Introduction: Many studies mention that variations in growth and orientation of the cranial base angle can alter the position of both jaws in relation to the cranial base. This study seeks to relate the cranial base angle according to skeletal classes in adult patients who attended the Dental Clinic of the Instituto Latinoamericano de Estudios Superiores in Asunción city in Paraguay, between 2010 and 2018, using lateral cephalometric radiographs. Materials and methods: Retrospective cross-sectional study. Skeletal classes were classified using the ANB angle and the NSBa angle of Varjanne and Koski to determine the cranial base angle. Chi-square tests were applied to associate the cranial base angle according to skeletal classes, with a confidence level of $95 \%$. Results: In this study, 185 lateral cephalometric radiographs were included in the study of people who attended the clinic. The mean values of NSBa angle were $128.84^{\circ} \pm 5.17^{\circ}$ for all classes; $128.27^{\circ} \pm 5.27^{\circ}$ for class I, $129.1^{\circ} \pm 4.7^{\circ}$ for class II and $127.84^{\circ} \pm 5^{\circ}$ for class III. The $65.95 \%$ of participants had NSBa angle values out of the norm, from which $87.7 \%$ reported a small cranial base angle. Conclusion: The average cranial base angle was found to be out of the norm in every skeletal class in decreasing order for classes III, I, and II, cranial flexure being more frequent than others.

Keywords: Orthodontics; skull base diagnostic imaging; cephalometry; Paraguay.

\section{Resumo}

Introdução: Numerosos estudos mencionam que as variações do crescimento e orientação do ângulo da base craniana podem alterar a posição de ambos os maxilares em relação com a base craniana. Este estudo busca relacionar o ângulo da base craniana com as classes esqueléticas, em telerradiografias laterais de pacientes adultos que acudiram à Clínica Odontológica do Instituto Latino-Americano de Estudos Superiores, da cidade de Asunción, Paraguai, nos anos 2010-2018. Materiais e métodos: estudo transversal retrospectivo. Para classificar a classe esquelética utilizou-se o ângulo ANB de Steiner e para determinar o ângulo da base craniana posterior o NSBa de Varjanne e Koski. Se aplicou a prova Qhi quadrado para relacionar o ângulo da base craniana e as classes esqueléticas, com um nível de confiança do 95 \%. Resultados: Formaram parte do estudo 185 telerradiografias laterais de crâneo de pacientes que acudiram à clínica. A média do ângulo NSBa foi $128.4^{\circ} \pm 5.17^{\circ}$; a classe I foi de $128.27^{\circ} \pm 5.27^{\circ}$, a classe II $129.1^{\circ} \pm 4.7^{\circ}$ e classe III $127.84^{\circ} \pm 5^{\circ}$. O $65.95 \%$ possui valores do ângulo NSBa fora da norma, dos quais o $87.7 \%$ apresentam um ângulo fechado. Conclusão: A média do ângulo da base craniana estava fora da norma em todas as classes esqueléticas, sendo mais frequente a flexão craniana, em ordem decrescente para as classes III, I e II.

Palavras-chave: ortodontia; base do Crâneo/diagnóstico por imagem; cefalometria; Paraguai. 


\section{Introducción}

Con de interés para el campo de la ortodoncia el crecimiento y desarrollo del complejo craneofacial, encontrándose en él una de las regiones más importantes en cuanto a su crecimiento, desarrollo, aspectos anatómicos y correlaciones faciales: la base del cráneo (1). Se debe tener en cuenta que la base del cráneo puede asumir dos modalidades de crecimiento: extensión (ángulo abierto) o flexión (ángulo cerrado) (2).

Durante el desarrollo, la base craneal anterior y posterior se flexionan en la silla turca en el plano sagital medio y, por lo tanto, constituyen un ángulo en la base craneal, denominado ángulo de la base craneal o ángulo de la silla de montar (3). Al nacer, este ángulo mide $142^{\circ}$ y disminuye a $132^{\circ}$ a la edad de 5 años. Desde los 5 años hasta los 15 años, el ángulo NasionSilla-Basion (NSBa) se vuelve relativamente estable (4-6). La sincondrosis esfeno-occipital se fusiona alrededor de los 18 años de edad $(7,8)$, y la longitud de la base posterior del cráneo aumenta en una dirección hacia atrás y hacia abajo debido al desplazamiento dorsal del punto Basion (Ba) (7). La media del rango de valor de NSBa es de $130^{\circ}$ a $135^{\circ}(9-13)$.

El complejo nasomaxilar se asocia con la base craneal anterior y la mandíbula con la base craneal posterior. Por lo tanto, es posible suponer, solo a partir de esta relación geométrica, que las variaciones en el crecimiento y la orientación de estas regiones pueden alterar las posiciones del maxilar y la mandíbula en relación con la base craneal y entre sí. Esto a su vez, según numerosos autores, puede influir en la clase esqueletal (2, 9, 11, 14-16). La base craneal desempeña un papel fundamental en el crecimiento craneofacial, al ayudar a integrar diferentes patrones de crecimiento en varias regiones contiguas del cráneo, como los componentes del cerebro, la cavidad nasal, la cavidad oral y la faringe, tanto funcional como espacialmente (17).

La variación en la expresión de diferentes patrones faciales es el resultado de la interacción de factores como la herencia, el ambiente y la función, que tienen un efecto sobre el crecimiento y el desarrollo del complejo maxilofacial (6).

Por otra parte, para la clasificación del patrón esquelético craneofacial puede emplearse el ángulo ANB de la cefalometría de Steiner. Este ángulo está formado por la intersección del plano NA con la línea de referencia SN. Este análisis relaciona la posición del maxilar superior con la base del cráneo (18).

En el ángulo abierto de la base craneal, la mandíbula se ubica en una posición retrusiva, dando como resultado una clase ósea II. Björk también establece que cualquier forma de la base craneal tendrá un efecto de desplazamiento de la cavidad glenoidea y, por consiguiente, de la protrusión o retrusión mandibular, es decir, ángulos abiertos corresponderán a clase II, ángulos cerrados a clase III y ángulos normales en clase I (19).

Existen numerosos estudios que han buscado describir o relacionar el ángulo de la base craneal con las clases esqueletales. Entre ellos, algunos sostienen que el ángulo de la base craneal 
no tiene ningún efecto sobre la formación de clases esqueletales, mientras que otros proponen lo contrario $(3,6,9-11,14,16-18,20-24,26)$.

Una revisión sistemática realizada por Almeida KCM, et al., en el año 2017, concluyó que “el ángulo de la base del cráneo no parece jugar un papel clave en el desarrollo de las clases esqueletales, siendo relativamente estable en edades de 5 a 15 años. Una flexión de la base del cráneo más obtusa, en asociación o no, con una mayor longitud de la base anterior del cráneo, puede contribuir al desarrollo de la clase II división II. Una flexión más aguda de la base del cráneo puede contribuir a un posicionamiento más anterior de la mandíbula y al desarrollo de una clase III también” (13). Es por esto que, teniendo en cuenta que no existen estudios publicados en la población paraguaya sobre el tema, se planteó como objetivo relacionar el ángulo de la base craneal en pacientes con diferentes clases esqueletales en telerradiografías de pacientes adultos que acudieron a la Clínica Odontológica del Instituto Latinoamericano de Estudios Superiores, en los años 2010 a 2018.

\section{Materiales y métodos}

C realizó un estudio transversal retrospectivo en el que se analizaron telerradiografías - laterales de pacientes de 18 a 60 años que acudieron a la Clínica Odontológica del Instituto Latinoamericano de Estudios Superiores (ILES) en la ciudad de Asunción, Paraguay, en los años 2010 a 2018. Se excluyeron radiografías de pacientes con tratamiento previo o actual de ortodoncia u ortopedia; aquellos con alteraciones craneofaciales, paladar fisurado y síndromes asociados. El tipo de muestreo fue no probabilístico de casos consecutivos.

Para la clasificación de la clase esqueletal se utilizó el ángulo ANB de Steiner y para la determinación del ángulo de la base craneal el ángulo NSBa de Varjanne y Koski (27). También se realizaron los calcos cefalométricos completos en papel vegetal, fijados con cinta de papel a las telerradiografías, con un portaminas de 0.5 milímetros, negatoscopio Cristófoli Luna ${ }^{\circledR}$ de luz fría con una luminosidad de 7000 Lux, en una habitación semioscura.

Una vez trazadas las estructuras anatómicas, se realizó la demarcación de los puntos craneales y anatómicos de interés, seguido del trazado de planos (figura 1) y mediciones de ángulos. Un mismo operador elaboró todos los calcos cefalométricos y trazados, y posteriormente, un experto en el área evaluó cada uno de ellos.

Puntos:

- A: punto más profundo de la curva del maxilar entre la espina nasal anterior y el borde del alvéolo.

- B: punto ubicado en la parte más profunda del contorno de la sínfisis mandibular en la zona alveolar. 
- $\mathrm{N}$ (Nasion): punto ubicado en el límite anterior de la sutura frontonasal.

- S (Silla): punto ubicado en el centro geométrico de la silla turca del hueso esfenoides.

- Ba (Basion): punto posteroinferior del hueso occipital en el margen anterior del foramen magno.

Planos:

- N-S: se traza desde el punto nasion al punto silla.

- S-Ba: se traza desde el punto silla al punto basion.

- N-A: se traza desde el punto nasion al punto A.

- N-B: se traza desde el punto nasion al punto B.

Ángulos:

- NSBa: está formado por la intersección de los planos N-S (base craneal anterior) con el plano S-Ba.

- ANB: está formado por la intersección de los planos NA y NB.

La clase esqueletal se evaluó según el ángulo ANB de Steiner modificado, teniendo en cuenta el rango de valores utilizados en el estudio de Polat et al. (21).

- Clase II ( $\geq 5)$.

- Clase III ( $\leq-1)$.

- Clase I (-0.5 a 4.5).

Para la selección de este rango se revisó un metaanálisis realizado por Gong et al. (28), en el que se pudo observar que la mayoría de los estudios que se incluían, utilizaban este rango de valor para determinar la clase esqueletal, de los cuales uno de ellos correspondía al estudio de Polat. Con respecto al rango del valor correspondiente a la clase I, se especificó de esa manera para no caer en confusiones puesto que en el rango $<5$ y $>-1$ se presentan valores que pueden redondearse.

Para determinar el ángulo de la base craneal se utilizó el ángulo NSBa por la cercanía del punto Ba a la base. Estos valores fueron clasificados de la siguiente manera (19):

- Ángulos normales $\left(130^{\circ}-135^{\circ}\right)$.

- Ángulos abiertos $\left(>135^{\circ}\right)$.

- Ángulos cerrados $\left(<130^{\circ}\right)$. 
Figura 1. Puntos y planos cefalométricos utilizados para el estudio.

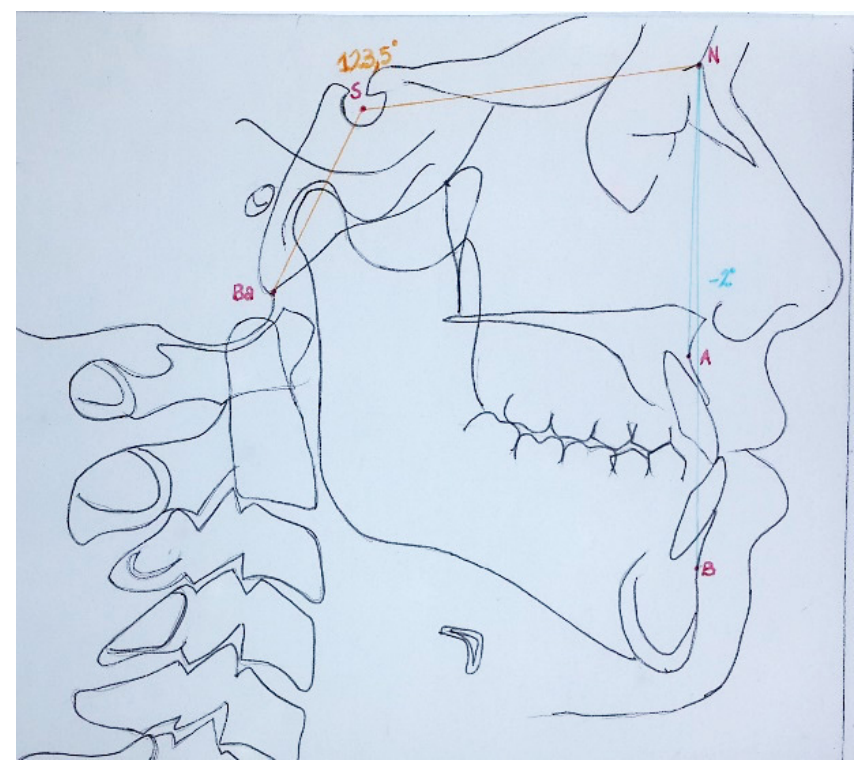

Fuente: elaboración propia.

Los datos se registraron y almacenaron en una planilla electrónica mediante el programa Microsoft Excel 2007. Los análisis se realizaron mediante el programa Epi Info ${ }^{\mathrm{TM}}$ 7. Se aplicó la prueba chi-cuadrado para relacionar el ángulo de la base craneal y las clases esqueletales, con un nivel de confianza del $95 \%$.

\section{Resultados}

Pra este estudio se usaron 185 telerradiografías laterales de pacientes adultos que acudieen Asunción, Paraguay, en los años 2010 a 2018. Se excluyeron 177 telerradiografías que no cumplieron con los criterios establecidos. La media de edad fue de 28 años \pm 7 .

En este estudio se pudo observar que la clase esqueletal más frecuente fue la clase I con el $47.57 \%$, seguido por la clase II con el $28.11 \%$ (figura 2 ). 
Figura 2. Clasificación de clase esqueletal en pacientes adultos que acudieron a la Clínica Odontológica del ILEs. Años 2010 a 2018.

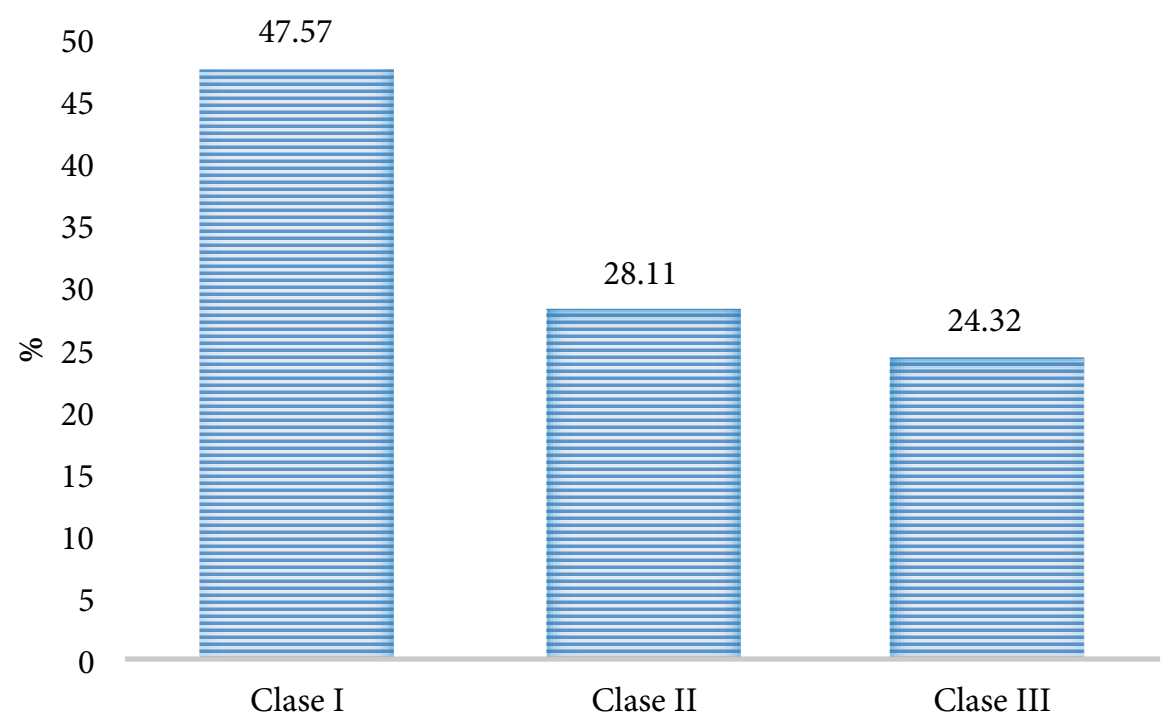

El 65.95\% de los pacientes presentó el ángulo de la base del cráneo fuera de la norma, de estos la mayoría tenía ángulo cerrado (figura 3).

Figura 3. Clasificación de clase esqueletal según valores de la base craneal fuera de los valores de la norma en pacientes adultos que acudieron a la Clínica Odontológica del iles. Años 2010 a 2018.

Nentro de la norma $\quad$ Fuera de la norma Angulo abierto $\quad$ Angulo cerrado

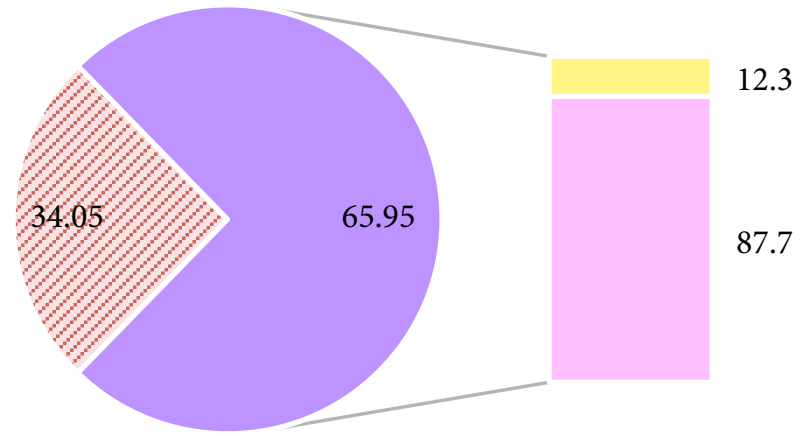

La media del ángulo NSBa fue $128.4^{\circ} \pm 5.17^{\circ}$; para la clase I fue de $128.27^{\circ} \pm 5.27^{\circ}$, para la clase II $129.1^{\circ} \pm 4.7^{\circ}$ y para la clase de III de $127.84^{\circ} \pm 5^{\circ}$ (tabla 1$)$. Al evaluar la asociación entre la clase esqueletal y el ángulo de la base craneal no se encontró relación estadísticamente significativa $(p=0.108)$. 
Tabla 1. Medidas de tendencia central y dispersión del ángulo de la base craneal según la clase esqueletal en pacientes adultos que acudieron a la Clínica Odontológica del iles. Años 2010 a 2018.

\begin{tabular}{cccccccccc}
\hline Clase & Nro. & Media & DE & Mínimo & P25 & Mediana & P75 & Máximo & Moda \\
\hline I & 88 & 128.27 & 5.27 & 112.5 & 124.75 & 128.00 & 131.75 & 141.00 & 130 \\
II & 52 & 129.10 & 4.71 & 117.5 & 126.00 & 129.25 & 133.00 & 139.00 & 126 \\
III & 45 & 127.84 & 5.49 & 118.00 & 125.00 & 128.00 & 130.00 & 145.50 & 128 \\
Total & 185 & 2.35 & 3.80 & -9.00 & -0.50 & 2.50 & 5.00 & 11.00 & 3
\end{tabular}

$D E=$ desviación estándar; $\mathrm{P}=$ Percentil 25 y 75. Valor de $\mathrm{p}=0.108$

La mayor distribución de los valores del ángulo de la base craneal en las tres clases esqueletales se encuentran fuera de la norma. De 88 pacientes clase I, solo 31 coincidieron con el ángulo normal (tabla 2).

Tabla 2. Distribución del ángulo de la base craneal por clase esqueletal con respecto a valores de la norma en pacientes adultos que acudieron a la Clínica Odontológica del ILEs. Años 2010 a 2018.

\begin{tabular}{cccc}
\hline & \multicolumn{2}{c}{ Ángulo de la base craneal con respecto a la norma } \\
& $\begin{array}{c}\text { Fuera } \\
\text { Nro. (\%) }\end{array}$ & $\begin{array}{c}\text { Dentro } \\
\text { Nro. (\%) }\end{array}$ & $\begin{array}{c}\text { Total } \\
\text { Nro. (\%) }\end{array}$ \\
\hline Clase esqueletal & & & \\
\hline I & $57(30.81)$ & $31(16.76)$ & $88(47.57)$ \\
II & $30(16.22)$ & $22(11.89)$ & $52(28.11)$ \\
III & $35(18.92)$ & $10(5.42)$ & $45(24.32)$ \\
Total & $122(65.95)$ & $163(88.11)$ & $185(100.00)$ \\
\hline
\end{tabular}

\section{Discusión}

S e realizó este trabajo para evaluar el ángulo de la base craneal según clases esqueletales, Sen telerradiografías laterales de pacientes adultos que acudieron a la Clínica Odontológica del Instituto Latinoamericano de Estudios Superiores (ILEs), en los años 2010 a 2018, encontrándose que no existe asociación entre el ángulo de la base del cráneo y la clase esqueletal, al igual que los estudios realizados en México, Rumania, Estados Unidos, Turquía, Reino Unido, India, Japón, Irak y Pakistán (6, 10, 12, 14, 17, 19, 20, 21, 23). En países como Alemania, China, Brasil, Francia y Perú, en cambio, se encontraron diferencias estadísticamente significativas $(9,11,16,24-26)$.

Con respecto a la distribución de sujetos según la clase esqueletal, en este estudio fue más frecuente la clase I ( $47 \%$ ), seguido por la clase II ( $28 \%$ ) y por último la clase III (24\%) de los 
sujetos, al contrario de otros estudios en los que la más predominante fue la clase II, como en aquellos que se realizaron en México (47.5\%), Perú (51.6\%), Japón (63\%) y Hong Kong, en el que la clase II fue ligeramente predominante (36.14\%) (11, 19, 22, 24). Sin embargo, los resultados de todos los estudios antes mencionados fueron similares a los de este, pues la clase III fue la menos predominante, a diferencia de lo encontrado en el estudio de Hong Kong, en el que las clases fueron cercanas en proporcionalidad en su distribución (24).

En cuanto al ángulo de la base del cráneo, en estudios realizados en Iraq y Brasil se encontraron valores normales dentro del rango $130^{\circ}$ a $135^{\circ}$, pero la media del ángulo NSBa en este estudio estuvo por debajo de dichos valores $\left(128.4 \pm 5.17^{\circ}\right)$, al igual que los valores encontrados en los estudios realizados en India $\left(128.84 \pm 11.2^{\circ}\right)$, Alemania $\left(129.7 \pm 4.7^{\circ}\right) \mathrm{y}$ Turquía $\left(126.95 \pm 4.98^{\circ}\right)(12,13,16,20,21)$. Sin embargo, el promedio obtenido en este estudio fue inferior a los obtenidos en otros estudios realizados en Perú (132 $\pm 4.85^{\circ}$ ), Reino Unido $\left(132.85 \pm 5.04^{\circ}\right)$, Pakistán $\left(130.9 \pm 5.3^{\circ}\right)$ Hong Kong $\left(130 \pm 4.9^{\circ}\right)$ y Rumania $\left(132.67 \pm 5^{\circ}\right)$ $(6,10,11,23,24)$.

En este estudio, el menor valor de la media del ángulo de la base craneal correspondió a la clase III, seguida de la clase I y, por último, la clase II, similar a los resultados obtenidos en Hong Kong, Rumania, Perú y Pakistán pero diferentes a los de Reino Unido en el que las clases I < III < II y Turquía en el que las clases III < II < I (6, 10, 11, 21, 23, 24).

Teniendo en cuenta el ángulo de la base craneal y al analizar la clasificación de la norma, en este estudio se encontró que la mayor parte de la muestra presentó valores fuera de la norma (65.95\%), ligeramente superior a lo encontrado en México (61.25\%) (19).

En cuanto a la distribución de sujetos según valores del ángulo de la base craneal, fuera de la norma en este estudio se observó que la mayor proporción correspondió al ángulo cerrado (87.7\%), lo que difiere del estudio realizado en México en el que el ángulo abierto fue predominante (85.7\%) (19).

Con respecto a la distribución de valores del ángulo de la base craneal según valores de la norma para cada clase esqueletal, en este estudio la mayor distribución se encontró fuera de la norma en las tres clases esqueletales ( $\mathrm{I}=64.5 \%$, II= 57.69 \% y III=77.78\%), observándose más valores dentro de la norma en pacientes clase II en relación con las otras clases esqueletales, mientras que en el estudio mexicano se observó que la mayor distribución de los valores también estuvo fuera de la norma en la clase I (60.71\%) y II (65.78\%), pero para la clase III este autor observó que el 50\% se encontraba dentro de la norma (19).

En este estudio se encontró que, de los valores fuera de la norma, el ángulo cerrado fue el más frecuente en las tres clases esqueletales, al predominar en las clases III (68.89\%), seguido de la clase I (55.68\%) y, por último, de la clase II (51.92\%). Se encontraron más valores dentro de la norma en pacientes clase II, en relación con las otras clases esqueletales (42.31\%). Sin embargo, en el estudio mexicano los valores fuera de la norma, tanto para la clase I (53.6 \%) como para la clase II (63.2\%) fueron más frecuentes los ángulos de la base craneal abiertos 
y para la clase III predominaron los ángulos cerrados (28.6\%). Björk establece que cualquier forma de la base craneal tendrá un efecto de desplazamiento de la cavidad glenoidea y, por consiguiente, de la protrusión o retrusión mandibular, es decir, ángulos abiertos corresponderán a la clase II, ángulos cerrados a la clase III y ángulos normales a la clase I (19).

Por otro lado, en este estudio se observó que, en la clase I esqueletal, una menor proporción de la muestra se ubicó dentro de los valores correspondientes a ángulos dentro de la norma (35.23\%), resultado similar al estudio mexicano (39.3\%). En la clase ósea II, solo una menor proporción de la muestra coincidió con los valores correspondientes a ángulos abiertos (5.77\%), diferente al estudio mexicano en el que una mayor proporción de la muestra coincidió con los valores correspondientes a dicho ángulo (63.2\%). En la clase III esqueletal, una mayor proporción de la muestra coincidió con los valores correspondientes ángulos cerrados (68.89\%), contrario al estudio mexicano en el que se observó una menor proporción (28.6\%) (19).

Con base en lo expuesto se puede considerar que es más probable encontrar, para los pacientes de la clase I, II y III, valores fuera de la norma, con predominio de los ángulos cerrados, en lugar de pacientes de clase I con valores dentro de la norma (130 a 135); de clase II con ángulos abiertos (mayores a 135); y de clase III con ángulos cerrados (menores a 130). En el momento de realizar un análisis cefalométrico no podríamos guiarnos por la regla mencionada anteriormente. Además, se puede afirmar que, al parecer, existe una influencia limitada del ángulo de la base como factor etiológico de la clase esqueletal, debido a que en este estudio no se encontró asociación entre el ángulo de la base y las clases esqueletales. El promedio del ángulo de la base craneal se encontró fuera de la norma en todas las clases esqueletales, siendo más frecuente la flexión craneal, en orden decreciente para las clases III, I y II.

\section{Contribución de los autores}

Cristell Amarilla: concepción, diseño, recolección y análisis de datos, redacción del
manuscrito.

Leticia Galeano: concepción y revisión crítica del manuscrito.

Clara Maldonado Renault: recolección y análisis de datos.

Julio Pratt Giosa: diseño e interpretación de resultados.

Clarisse Díaz-Reissner: análisis de datos, redacción del manuscrito y revisión crítica del manuscrito.

Todos los autores dieron su aprobación final y aceptan ser responsables de todos los aspectos del trabajo. 


\section{Conflicto de intereses}

$\mathrm{N}^{\text {manuorodectarata. }}$

\section{Referencias}

1. Toledo Jaramillo DT, Cazar Almache M, Bravo Calderón M. Correlación de la base del cráneo con el patrón facial y la posición sagital de los maxilares. Rev Odonlog Lat Ortod y Odontoped. 2014;48(3):1-18.

2. Sato, S. The masticatory organ: functions and dysfunctions. En: Slavicek R, editor. The dynamic functional anatomy of the craniofacial complex its and relation to the articulations of dentition. Edición única. Klosterneuburg, Austria: GAMMA MedizinischWissenschaftliche Fortbildung-AG; 2002. p. 484-90.

3. Cendekiawan T, Wong RWK, Rabie ABM. Relationships between cranial base synchondroses and craniofacial development: A review. Open Anat J. 2010;2(1):67-75. Doi: $10.2174 / 1877609401002010067$

4. Arat M, Köklü A, Ozdiler E, Rübendüz M, Erdoğan B. Craniofacial growth and skeletal maturation: a mixed longitudinal study. Eur J Orthod. 2001;23(4):355-361.

5. Arat ZM, Rübendüz M, Akgül AA. The displacement of craniofacial reference landmarks during puberty: a comparison of three superimposition methods. Angle Orthod. 2003;73(4):374-380. Doi: 10.1043/0003-3219(2003)073<0374:TDOCRL>2.0.CO;2

6. Shah R, Mushtaq M, Mahmood A. The relationship between cranial base angle and various malocclusion types. Pak Orthod J. 2015;7(1):8-12.

7. Fathi H, Mohammad-Rabei E, Kabiri S, Baghban AA, Soheilifar S, Nouri M. Craniofacial and airway growth in 9-11 years old normal dental occlusion in Iranian adolescents: A longitudinal cephalometric study. Dent Hypotheses. 2018;8(1):8-16. Doi: 10.4103/denthyp.denthyp_55_16

8. Gregoret J. Ortodoncia y cirugía ortognática: diagnóstico y planificación. 2da. Ed. Caracas: AMOLCA; 2014.

9. Dias de Almeida Berger OP, Menezes Marchioro E, Deon Rizzato SM, Martinelli Santayana de Lima E. Comparative study of linear and angular measures of the cranial base in skeletal Class I and III malocclusion. Rev Odonto Ciênc. 2011;26(2):126-132. Doi: 10.1590/ S1980-65232011000200006

10. Dhopatkar A, Bhatia S, Rock P. An investigation into the relationship between the cranial base angle and malocclusion. Angle Orthod. 2002;72(5):456-463. Doi: 10.1043/0003-3219(2002)072<0456:AIITRB >2.0.CO;2

11. Flores Angeles A, Soldevilla Galarza L. Evaluación de la deflexión de la base del cráneo. Un estudio cefalométrico. Odontol Sanmarquina. 2017;20(1):23-26. Doi: 10.15381/ os.v20i1.13546 
12. Abd BI, Ali FA. Cranial base morphology in different skeletal classes (A cross-sectional lateral cephalometric study). J Baghdad Coll Dent. 2013;25(Special Is 1):108-113.

13. Almeida KCM, Raveli TB, Vieira CIV, et al. Influence of the cranial base flexion on Class I, II and III malocclusions: a systematic review. Dent Press J Orthod. 2017;22(5):56-66. Doi: 10.1590/2177-6709.22.5.056-066.oar

14. Andria LM, Leite LP, Prevatte TM, King LB. Correlation of the cranial base angle and its components with other dental/skeletal variables and treatment time. Angle Orthod. 2004;74(3):361-366. Doi: 10.1043/0003-3219(2004)074<0361:COTCBA>2.0.CO;2

15. Vandekar M, Kulkarni P, Vaid N. Role of cranial base morphology in determining skeletal anteroposterior relationship of the jaws. J Indian Orthod Soc. 2013;47(5):245-248. Doi: 10.5005/jp-journals-10021-1167

16. Proff P, Will F, Bokan I, Fanghänel J, Gedrange T. Cranial base features in skeletal class III patients. Angle Orthod. 2008;78(3):433-439. Doi: 10.2319/013007-48.1

17. Agarwal A, Pandey H, Bajaj K, Pandey L. Changes in cranial base morphology in class I and class II Division 1 malocclusions. J Int Oral Health. 2013;5(1):39-42.

18. Barrachina Mataix C. Cefalometría. En: Canut Brusola JA, editor. Ortodoncia clínica y terapeútica. 2da. Ed. Barcelona: Masson; 2005:174-202.

19. Ruiz Rivera AJ, Canseco Jiménez J, Cuairán Ruidíaz V. Relación entre la deflexión de la base del cráneo y la clase ósea. Rev Odont Mex. 2011;15(4):214-218.

20. Agrawal M, Mehta F, Mehta S. An investigation into the relationship between the cranial base angle and malocclusion in gujarati population. Indian J Basic Appl Med Res. 2014;4(1):324-348.

21. Polat Ö, Kaya B. Changes in cranial base morphology in different malocclusions. Orthod Craniofac Res. 2007;10(4):216-221. Doi: 10.1111/j.1601-6343.2007.00403.x

22. Kasai K, Moro T, Kanazawa E, Iwasawa T. Relationship between cranial base and maxillofacial morphology. Eur J Orthod. 1995;17(5):403-410. Doi: 10.1093/ejo/17.5.403

23. Panainte I, Suciu V, Mártha K-I. Original research. Correlation between cranial base morphology and various types of skeletal anomalies. J Interdiscip Med. 2017;2(S1):5761. Doi: 10.1515/jim-2017-0007

24. Chin A, Perry S, Liao C, Yang Y. The relationship between the cranial base and jaw base in a Chinese population. Head Face Med. 2014;10(1):31. Doi: 10.1186/1746-160X-10-31

25. Bacon W, Eiller V, Hildwein M, Dubois G. The cranial base in subjects with dental and skeletal Class II. Eur J Orthod. 1992;14(3):224-228. Doi:10.1093/ejo/14.3.224

26. Alves PV, Mazuchelli J, Patel PK, Bolognese AM. Cranial base angulation in Brazilian patients seeking orthodontic treatment. J Craniofac Surg. 2008;19(2):334-338. Doi: 10.1097/ SCS.0b013e3181539b70

27. Qadir M. To evaluate the association between cranial base angle and malocclusions in sagittal plane. International Journal of Advance Research, Ideas and Innovations in Technology. 2017;3(5):496-500.

28. Gong A, Li J, Wang Z, et al. Cranial base characteristics in anteroposterior malocclusions: A meta-analysis. Angle Orthod. 2016;86(4):668-680. Doi: 10.2319/032315-186.1 